\title{
A ÉTICA PROFISSIONAL NA FORMAÇÃO DE PSICÓLOGOS: UM ESTUDO BIBLIOGRÁFICO E DOCUMENTAL ${ }^{1}$
}

\author{
Juliene de Cassia LEIVA ${ }^{2}$ \\ Sebastião de Souza LEMES $^{3}$
}

Resumo: Foi realizado um estudo bibliográfico na base de dados Scielo, de natureza descritiva, de modo a conhecer como o tema ética profissional se faz presente na formação de psicólogos. Os dados obtidos foram analisados de forma articulada a documentos subsidiários dessa formação, como as Diretrizes Nacionais para a Formação de Psicólogos e o Código de Ética Profissional do Psicólogo, apontando para os principais desafios a serem considerados na atualidade, em tal contexto. Foi também analisado o Projeto Pedagógico do curso de Psicologia de um Centro Universitário do interior do Estado de São Paulo, que evidenciou a operacionalização dos eixos estruturantes propostos pelas diretrizes, os quais abrangem parcialmente o componente "ética profissional", com ênfase em aspectos procedimentais e atitudinais na formação do psicólogo.

Palavras-chave: Ética profissional. Formação em psicologia. Diretrizes curriculares.

\section{INTRODUÇÃO}

O presente estudo se propõe a refletir sobre a inserção do tema ética profissional na formação em Psicologia. Tal propósito se justifica a partir das considerações acerca da peculiaridade da dimensão ética, envolvida na compreensão e no trabalho desse profissional. Tendo em vista as implicações éticas da atuação do psicólogo, frente aos desafios inerentes à sua profissão, chamam a atenção os "princípios fundamentais", apresentados no Código de Ética Profissional do Psicólogo, elaborado pelo Conselho Federal de Psicologia (2005), que se encontram entre os documentos que subsidiam a orientação profissional em sua formação. Além de considerar o conteúdo do Código de Ética Profissional do Psicólogo, é relevante observar também as indicações das Diretrizes Curriculares Nacionais para a Formação de Psicólogos (BRASIL, 2011). Tais

1 Trabalho realizado na pesquisa "A equiparação de oportunidades em discussão: implicações do financiamento estudantil no âmbito do ensino superior”, sob orientação do Prof. Dr. Sebastião de Souza Lemes, no Doutorado do Programa de PG em Educação Escolar da FCL-CAr UNESP.

${ }^{2}$ Psicóloga. UNESP - Universidade Estadual Paulista - Campus Assis. Mestre em Educação Especial pela UFSCar. Doutoranda em Educação Escolar na UNESP - Universidade Estadual Paulista - Campus Araraquara. Araraquara - SP - Brasil. 14800-901 - julieneleiva@ gmail.com.

${ }^{3}$ Doutor em Psicologia pelo Instituto de Psicologia. USP - Universidade de São Paulo. Professor da UNESP - Universidade Estadual Paulista - Campus Araraquara. Araraquara - SP - Brasil. 14800-901 ss.lemes2@gmail.com 
diretrizes indicam a obrigatoriedade de que a graduação em Psicologia esteja organizada em função dos chamados "eixos estruturantes", a saber: fundamentos epistemológicos e históricos; fundamentos teórico-metodológicos; procedimentos para a investigação científica e a prática profissional; fenômenos e processos psicológicos; interfaces com campos afins do conhecimento e práticas profissionais. Os eixos estruturantes definidos no documento traduzem as tendências a serem absorvidas nos projetos pedagógicos dos cursos de graduação em Psicologia, ainda que com algumas variações. Nesse sentido, é importante que sejam consideradas e constantemente revistas, com o intuito de refletir acerca das prioridades e compromissos assumidos no contexto mais amplo da formação profissional.

\section{Objetivo}

Refletir acerca da inserção e desenvolvimento do tema "ética profissional" na formação de psicólogos, considerando a produção bibliográfica da base de dados SciELO de 2010 a julho de 2015, bem como documentos subsidiários à graduação em Psicologia e à atuação dos profissionais dessa área frente a questões ligadas à ética profissional, e em particular o Projeto Pedagógico do Curso de Psicologia em um Centro Universitário do interior do Estado de São Paulo.

\section{Metodologia}

Foi realizado um levantamento bibliográfico na base de dados SciELO, fazendo uso de expressões de busca relacionadas ao interesse deste estudo. Foram selecionados e analisados artigos publicados a partir de 2010 até julho de 2015. Foi realizado ainda um estudo do Projeto Pedagógico do Curso de Psicologia de um Centro Universitário do interior do Estado de São Paulo, considerando os planos de ensino das disciplinas existentes no currículo, em termos de sua pertinência quanto aos eixos estruturantes estabelecidos nas referidas Diretrizes o que permitiu discutir, em linhas gerais, aspectos significativos de sua operacionalização.

\section{Resultados e discussão}

O levantamento bibliográfico realizado mostra a ocorrência de um número significativo de estudos sobre a "educação superior" e o "ensino superior", como também se destacam os relativos a "graduação", "ética" e "universidade", embora seja relativamente baixo o número de estudos, entre eles, que abordem a formação em 
Psicologia e, ainda que a inserção do tema "ética profissional”, o que evidencia a lacuna de conhecimento na área.

A partir da expressão de busca “educação superior", são seis os artigos que vem ao encontro do objetivo deste estudo. Os mais recentes, de Dalbosco (2015) e Goergen (2014), discutem acerca do conflito existente entre as pressões para o ensino predominantemente técnico/tecnológico e a necessidade de uma formação humanista no nível superior. Souto, Batista e Alves-Batista (2014), abordam a chamada educação interprofissional, no preparo para a atuação colaborativa, voltada para o cuidado integral em saúde, prevista nas Diretrizes (BRASIL, 2011). O artigo de Seixas et al. (2013) aponta como tais diretrizes podem ser contempladas no projeto pedagógico do curso de Psicologia, tendo em vista as peculiaridades da formação nessa área. Os artigos de Finkler et al. (2010) e de Finkler, Caetano e Ramos (2012) destacam a formação ética do profissional da saúde, enquanto decisiva para a sua atuação nos mais diferentes contextos. Conforme os autores, as estratégias de ensino das habilidades envolvidas nessa formação devem ser planejadas cuidadosamente, considerando sua importância no contexto mais amplo da atuação profissional. Com a expressão de busca "formação dos profissionais de saúde”, foi encontrado um artigo que atende aos critérios estabelecidos, de Lerner et al. (2013). O autor aborda a contribuição do psicólogo em sua atuação no sistema de saúde pública, garantindo o cuidado com o desenvolvimento humano, de forma integral.

A expressão de busca "formação em psicologia" permitiu o acesso ao artigo de Lima e Souza (2014), que vem ao encontro do interesse deste estudo, na medida em que indica a necessidade de que as Diretrizes Curriculares sejam discutidas quanto ao tipo de psicólogo que pretendem formar, ao sugerirem uma tendência para o atendimento interprofissional, frente às demandas da atualidade. $\mathrm{O}$ artigo de Macedo e Dimenstein (2011), encontrado a partir da expressão de busca "formação profissional do psicólogo", evidencia o fato de que muitas vezes o currículo na formação do psicólogo não se mostra coerente com os avanços ocorridos na produção de conhecimento na área, especialmente em se tratando de saúde mental, em que o atendimento oferecido ainda se baseia predominantemente no modelo clínico em contraposição ao modelo asilar. Com a expressão "ensino superior", foram encontrados os trabalhos de Bernardes (2012) e o de Vilela (2012) ainda sobre o currículo na formação do psicólogo, bem como o artigo de Santos et al. (2011), e o de Carneiro (2010), enfatizando a importância do ensino de Ética nos cursos da área da saúde. 
$\mathrm{O}$ artigo mais recente encontrado a partir da expressão de busca "ética" foi o de Silva (2015), que identificou como principais valores de estudantes de Psicologia de uma Universidade Pública os itens "dinheiro" e "fama”, o que é incompatível com os princípios do Código de Ética Profissional do Psicólogo (CONSELHO FEDERAL DE PSICOLOGIA, 2005). Apenas um artigo, entre os encontrados a partir de tal expressão de busca, refere-se a questões éticas ligadas à pesquisa em Psicologia (BORGES et al., 2013), o que denota a incipiente discussão a esse respeito no no Brasil. Amendola (2014) e Benelli (2014) atentam para as demandas sociais existentes na atualidade, perante as quais a formação ética se evidencia. Gerber e Zagonel (2013) destacam a ética enquanto aspecto fundamental da formação superior na área da saúde, em sua perspectiva integrativa. Rasera, Oliveira e Jesus (2014) reafirmam tal importância, porém discutem suas implicações nas condições de trabalho encontradas pelos psicólogos. Zotareli et al. (2012) aborda a violência de gênero entre estudantes universitários, enquanto Teixeira (2010) e Toledo e Pinafi (2012) evidenciam o papel do psicólogo frente a questões envolvendo a diferenciação de gênero. Maesso (2014), Miranda e Campos (2013), Souza e Coelho (2012), Dassoler e Palma (2012) e CostaRosa (2011) discutem a dimensão ética da abordagem psicanalítica do profissional da psicologia.

Com a expressão de busca "ética profissional", foram encontrados 3 artigos que abordam a atuação do psicólogo (ROCHA et al., 2015; DE MARTINI, 2011; FUENTES-ROJAS, 2011), voltados para a participação desse profissional nas equipes de trabalho, em situações tão diversas como o âmbito do atendimento em saúde e em educação, fortemente marcados pela demanda por cuidados do psicólogo. O artigo de Oliveira, Ayres e Zoboli (2011), discutem a postura ética do psicólogo ao atuar em situações críticas. La Taille (2010), por sua vez, estuda a dimensão psicológica do desenvolvimento moral, intimamente relacionado à capacidade de reflexão e de autonomia do sujeito em relação às decisões sobre si mesmo diante dos outros, englobando o necessário embasamento teórico presente na formação em Psicologia.

Com a expressão de busca "formação em recursos humanos", o artigo de Berger (2011) mostra as peculiaridades a serem consideradas pelos profissionais durante o atendimento, o que está diretamente relacionado à qualidade de sua formação. A expressão de busca "formação do psicólogo" conduziu ao artigo de Melo e Barreto (2014) e aos trabalhos de Poppe e Batista (2012) e de Brasileiro e Souza (2010), que discutem a formação do psicólogo considerando a organização curricular existente e os 
desafios que esse profissional tem a enfrentar. A partir da expressão de busca "universidade", foram encontrados três artigos voltados para a importância do envolvimento em situações de estágio e de supervisão clínica, enquanto componentes da formação em psicanálise, em termos éticos e técnicos (DERZI; MARCOS, 2013; DARRIBA, 2011; MARCOS, 2011; PINHEIRO; DARRIBA, 2010). Do cruzamento entre os termos de busca "ética" e "Psicologia", foram encontrados três artigos, dos quais dois (ALVARENGA, 2012; VAHLE, 2011) se aplicam ao interesse deste estudo. O primeiro trabalho aborda os dilemas éticos enfrentados por psicólogos, envolvendo a questão do sigilo profissional. O segundo discute a dimensão moral na concepção freudiana do sujeito, enquanto fundamentação teórica indispensável na formação do psicólogo. A lacuna quanto à produção de conhecimento sobre a Ética Profissional indica a necessidade de maior atenção por parte dos pesquisadores interessados em compreender a formação de profissionais da Psicologia e áreas afins, considerando especialmente os determinantes e as implicações dessa lacuna em termos de atuação profissional.

Além disso, a análise do Projeto Pedagógico do Curso de Psicologia de um Centro Universitário do interior do Estado de São Paulo permitiu observar como a organização das disciplinas do curso traduz a proposta das Diretrizes Curriculares Nacionais para a Formação de Psicólogos, evidenciando a pertinência dessas disciplinas em relação aos chamados eixos estruturantes de tal formação. Com base na análise realizada, foi possível notar que as disciplinas que correspondem aos eixos "fundamentos teórico-metodológicos" (com ênfase nos aspectos metodológicos) e "práticas profissionais", ligados mais diretamente à atuação profissional, são as que existem em maior quantidade e ocupam a maior parte do tempo destinado à graduação em Psicologia, em detrimento das disciplinas ligadas à produção de conhecimento e aos fundamentos históricos e epistemológicos da área de estudo. A existência de tal ênfase confirma o que a literatura na área tem mencionado, ao apontar para a tendência do ensino superior de voltar-se para os interesses de mercado, valorizando assim os aspectos predominantemente técnicos da atuação profissional. Enquanto componente curricular, a Ética Profissional destaca-se por apresentar uma carga horária reduzida (40 horas), em relação ao total do curso (4500 horas), sendo previsto no Projeto Pedagógico em questão que deverá ser explorada nas demais disciplinas. Assim, a "ética profissional" não está circunscrita a um eixo estruturante apenas, mas perpassa todos 
eles, revelando, desse modo, a ênfase procedimental e atitudinal que vem a ser atribuída a tal dimensão do trabalho do psicólogo.

\section{Considerações finais}

Com base no levantamento bibliográfico realizado, evidenciou-se a lacuna significativa existente na literatura acerca da realidade estudada. Faz-se necessário intensificar a produção de conhecimento na área, de modo a mobilizar a reflexão e o debate sobre a educação superior e seus desdobramentos, particularmente em se tratando de profissionais que podem atuar em variadas frentes de trabalho na promoção do desenvolvimento humano. A análise do Projeto Pedagógico do Curso, por sua vez, evidenciou a operacionalização dos eixos estruturantes propostos nas Diretrizes Nacionais para a Formação de Psicólogos. Evidenciou ainda o fato de que a "Ética Profissional" do psicólogo, enquanto conjunto de conceitos, procedimentos e atitudes, perpassa todos os eixos propostos.

THE PROFESSIONAL ETICS ON THE PSYCOLOGISTS FORMATION: A
BIBLIOGRAPHICAL AND DOCUMENTAL STUDY

Abstract: A literature study was conducted in Scielo database, descriptive, in order to know how professional ethics theme is present in the training of psychologists. Data were analyzed in an articulated manner to ancillary documents such training as the National Guidelines for Training Psychologists and Code of Professional Ethics of Psychologist, pointing to the main challenges to be considered today, in such a context. It was also analyzed the Educational Psychology Course Design of a University Center in the state of São Paulo, which showed the operationalization of the structuring axes proposed by the guidelines, which partially cover the component "professional ethics", with emphasis on procedural aspects and attitudinal the training of psychologists.

Key words: Professional ethics. Training in psychology. Curriculum guidelines.

\section{REFERÊNCIAS}

ALVARENGA, P. et al. Questões éticas da pesquisa em Psicologia do Desenvolvimento. Psicologia: ciência e profissão, Brasília, v.32, n.4, p.856-871, 2012. Disponível em: <http://www.scielo.br/scielo.php?script=sci_arttext\&pid=S141498932012000400007\&lng=en\&nrm=iso>. Acesso em: 22 jul. 2015. 
AMENDOLA, M. F. Formação em psicologia, demandas sociais, contemporâneas e ética: uma perspectiva. Psicologia: ciência e profissão, Brasília, v.34, n.4, p.971-983, dec. 2014. Disponível

<http://www.scielo.br/scielo.php?script=sci_arttext\&pid=S141498932014000400971\&lng=en\&nrm=iso>. Acesso em: 22 jul. 2015.

BENELLI, S. J. As éticas nas práticas de atenção psicológica na assistência social. Estudos de Psicologia (Campinas), Campinas, v.31, n.2, p.269-287, jun. 2014. Disponível em: <http://www.scielo.br/scielo.php?script=sci_arttext\&pid=S0103166X2014000200012\&lng=en\&nrm=iso>. Acesso em: 22 jul. 2015.

BERGER, S. M. D. Violência entre parceiros íntimos: desafios no ensino e atenção em saúde. Revista Brasileira de Educação Médica, Rio de Janeiro, v.35, n.4, p.526-534, dez. 2011. Disponível em: $<$ http://www.scielo.br/scielo.php?script=sci_arttext\&pid=S010055022011000400012\&lng=en\&nrm=iso>. Acesso em: 22 jul. 2015.

BERNARDES, J. S. A formação em psicologia após 50 anos do Primeiro Currículo Nacional da Psicologia: alguns desafios atuais. Psicologia: ciência e profissão, Brasília, v.32, n.spe, p.216-231, 2012. Disponível em: $<$ http://www.scielo.br/scielo.php?script=sci_arttext\&pid=S141498932012000500016\&lng=en\&nrm=iso>. Acesso em: 22 jul. 2015.

BORGES, L. S. et al. Abordagens de gênero e sexualidade na Psicologia: revendo conceitos, repensando práticas. Psicologia: ciência e profissão, Brasília, v.33, n.3, p.730-745, 2013. Disponível em: $<$ http://www.scielo.br/scielo.php?script=sci_arttext\&pid=S141498932013000300016\&lng=en\&nrm=iso>. Acesso em: 22 jul. 2015.

BRASIL. Ministério da Educação. Diretrizes Curriculares Nacionais para a Formação de Psicólogos. Brasília: Ministério da Educação, 2011. Disponível em: <file:///C:/Users/User/Downloads/rces005_11\%20(1).pdf >. Acesso em: 22 jul. 2015.

BRASILEIRO, T. S. A.; SOUZA, M. P. R. Psicologia, diretrizes curriculares e processos educativos na Amazônia: um estudo da formação de psicólogos. Psicologia Escolar e Educacional, Campinas, v.14, n.1, p.105-120, jun. 2010. Disponível em: <http://www.scielo.br/scielo.php?script=sci_arttext\&pid=S1413$85572010000100012 \& \operatorname{lng}=$ en\&nrm=iso>. Acesso em: 22 jul. 2015.

CARNEIRO, L. A. et al. O ensino da ética nos cursos de graduação da área de saúde. Revista Brasileira de Educação Médica, Rio de Janeiro, v.34, n.3, p.412421, set. 2010. Disponível em: $<$ http://www.scielo.br/scielo.php?script=sci_arttext\&pid=S0100$55022010000300011 \& \operatorname{lng}=$ en\&nrm=iso>. Acesso em: 22 jul. 2015.

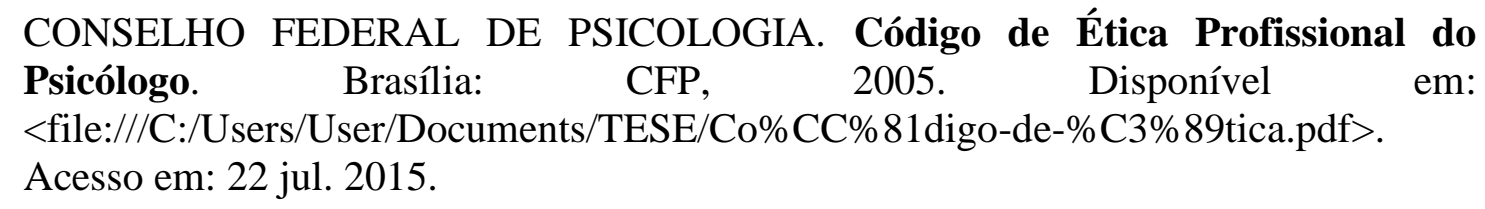


COSTA-ROSA, A. Ética e clínica na atenção psicossocial: contribuições da psicanálise de Freud e Lacan. Saúde e sociedade, São Paulo, v.20, n.3, p.743-757, set. 2011.

Disponível

em:

<http://www.scielo.br/scielo.php?script=sci_arttext\&pid=S0104-

12902011000300018\&lng=en\&nrm=iso>. Acesso em: 22 jul. 2015.

DALBOSCO, C. A. Educação superior e os desafios da formação para a cidadania democrática. Avaliação: Revista da Avaliação da Educação Superior, Sorocaba, v.20, n.1, 2015. Disponível em: <http://submission.scielo.br/index.php/aval/article/view/110174>. Acesso em: 22 jul. 2015.

DARRIBA, V. A. O lugar do saber na psicanálise e na universidade e seus efeitos na experiência do estágio nas clínicas-escola. Ágora: Estudos em teoria psicanalista, Rio de Janeiro, v.14, n.2, p.293-306, dec. 2011. Disponível em: $<$ http://www.scielo.br/scielo.php?script=sci_arttext\&pid=S 151614982011000200009\&lng=en\&nrm=iso>. Acesso em: 22 jul. 2015.

DASSOLER, V. A.; PALMA, C. M. S. A dimensão da ética nas intervenções do analista frente às demandas institucionais dos CAPS. Revista Latinoamericana de Psicopatologia fundamental, São Paulo, v.15, n.1, p.94-107, mar. 2012. Disponível em: <http://www.scielo.br/scielo.php?script=sci_arttext\&pid=S141547142012000100008\&lng=en\&nrm=iso>. Acesso em: 22 jul. 2015.

DE MARTINI, A. Reabilitação, ética e técnica. Ciência \& Saúde coletiva, Rio de Janeiro, v.16, n.4, p.2263-2269, abr. 2011. Disponível em: $<$ http://www.scielo.br/scielo.php?script=sci_arttext\&pid=S1413-

$81232011000400025 \& \operatorname{lng}=e n \& n r m=$ iso >. Acesso em: 22 jul. 2015.

DERZI, C.; MARCOS, C. M. Supervisão em psicanálise na universidade. Psicologia em Estudo, Maringá, v.18, n.2, p.323-331, jun. 2013. Disponível em: $<$ http://www.scielo.br/scielo.php?script=sci_arttext\&pid=S1413-

73722013000200013\&lng=en\&nrm=iso>. Acesso em: 22 jul. 2015.

FINKLER, M. et al. Formação profissional ética: um compromisso a partir das diretrizes curriculares? Trabalho, Educação e Saúde, Rio de Janeiro, v.8, n.3, p.449462, nov. 2010. Disponível em: $<$ http://www.scielo.br/scielo.php?script=sci_arttext\&pid=S1981$77462010000300007 \& \operatorname{lng}=\mathrm{en} \& n \mathrm{~nm}=\mathrm{iso}>$. Acesso em: 22 jul. 2015.

FINKLER, M.; CAETANO, J. C.; RAMOS, F. R. S. O cuidado ético-pedagógico no processo de socialização profissional: por uma formação ética. Interface Comunicação, Saúde e Educação, Botucatu, 2012. Disponível em: <http://www.scielo.br/pdf/icse/2012nahead/aop5012.pdf〉. Acesso em: 22 jul. 2015.

FUENTES-ROJAS, M. Psicologia e saúde: a terapia comunitária como instrumento de sensibilização para o trabalho com comunidades na formação do Psicólogo. Psicologia: ciência e profissão. Brasília, v.31, n.2, p.420-439, 2011. Disponível em: $\quad<$ http://www.scielo.br/scielo.php?script=sci_arttext\&pid=S141498932011000200016\&lng=en\&nrm=iso>. Acesso em: 22 jul. 2015. 
GERBER, V. K. Q.; ZAGONEL, I. P. S. A ética no ensino superior na área da saúde: uma revisão integrativa. Revista Bioética, Brasília, v.21, n.1, p.168-178, abr. 2013.

Disponível

<http://www.scielo.br/scielo.php?script=sci_arttext\&pid=S1983-

80422013000100020\&lng=en\&nrm=iso>. Acesso em: 22 jul. 2015.

GOERGEN, P. Tecnociência, pensamento e formação na educação superior. Avaliação: Revista da Avaliação da Educação superior (Campinas), Sorocaba, v.19, n.3, p.561-584, nov. 2014. Disponível em: $<$ http://www.scielo.br/scielo.php?script=sci_arttext\&pid=S1414-

$40772014000300003 \& \operatorname{lng}=$ en\&nrm=iso>. Acesso em: 15 jul. 2015.

LA TAILlE, Y. Moral e ética: uma leitura psicológica. Psicologia: teoria e pesquisa, Brasília, v.26, n.spe, p.105-114, 2010. Disponível em: $<$ http://www.scielo.br/scielo.php?script=sci_arttext\&pid=S010237722010000500009\&lng=en\&nrm=iso>. Acesso em: 12 jul. 2015.

LERNER, R. et al. A Psicologia na articulação entre os âmbitos coletivo e psíquico: construção de uma política pública em saúde de cuidado com o desenvolvimento. Psicologia: ciência e profissão, Brasília, v.33, n.spe, p.100-111, $2013 . \quad$ Disponível <http://www.scielo.br/scielo.php?script=sci_arttext\&pid=S141498932013000500011\&lng=en\&nrm=iso>. Acesso em: 12 jul. 2015.

LIMA, V. C.; SOUZA, R. T. Formação humana e competências: o debate nas diretrizes curriculares de psicologia. Psicologia \& Sociedade, Belo Horizonte, v.26, n.3, p.792-802, dez. 2014. Disponível em: $<$ http://www.scielo.br/scielo.php?script=sci_arttext\&pid=S0102-

$71822014000300027 \& \operatorname{lng}=$ en\&nrm=iso>. Acesso em: 13 jul. 2015.

MACEDO, J.; DIMENSTEIN, M. Formação do psicólogo para a saúde mental: a psicologia piauiense em análise. Interface - Comunicação, Saúde e Educação, Botucatu, v.15, n.39, p.1145-1158, dez. 2011. Disponível em: <http://www.scielo.br/scielo.php?script=sci_arttext\&pid=S141432832011000400014\&lng=en\&nrm=iso>. Acesso em: 13 jul. 2015.

MAESSO, M. C. A razão do diagnóstico em torno do ideal científico e a ruptura ética da psicanálise. Psicologia: teoria e pesquisa, Brasília, v.30, n.4, p.433-439, dez. $2014 . \quad$ Disponível em: <http://www.scielo.br/scielo.php?script=sci_arttext\&pid=S010237722014000400008\&lng=en\&nrm=iso>. Acesso em: 22 jul. 2015.

MARCOS, C. M. Reflexões sobre a clínica-escola, a psicanálise e sua transmissão. Psicologia clínica, Rio de Janeiro, v.23, n.2, p.205-220, 2011. Disponível em: $<$ http://www.scielo.br/scielo.php?script=sci_arttext\&pid=S010356652011000200013\&lng=en\&nrm=iso>. Acesso em: 14 jul. 2015.

MELO, R. A.; BARRETO, D. J. Formação em psicologia: discursos e saberes sobre experimentações de gênero. Psicologia: ciência e profissão, Brasília, v.34, n.3, p.676-689, set. 2014. Disponível em: 
<http://www.scielo.br/scielo.php?script=sci_arttext\&pid=S1414-

98932014000300676\&lng=en\&nrm=iso>. Acesso em: 20 jul. 2015.

MIRANDA, L.; CAMPOS, R. T. O. Balizamentos éticos para o trabalho em saúde mental: uma leitura psicanalítica. Revista Latinoamericana de Psicopatologia Fundamental, São Paulo, v.16, n.1, p.100-115, mar. 2013. Disponível em: $<$ http://www.scielo.br/scielo.php?script=sci_arttext\&pid=S141547142013000100008\&lng=en\&nrm=iso>. Acesso em: 22 jul. 2015.

OLIVEIRA, L. A.; AYRES, J. R. C. M.; ZOBOLI, E. L. C. P. Conflitos morais e atenção à saúde em Aids: aportes conceituais para uma ética discursiva do cuidado. Interface - Comunicação, Saúde e Educação, Botucatu, v.15, n.37, p.363-75, abr./jun. 2011.

PINHEIRO, N. N. B.; DARRIBA, V. A. A clínica psicanalítica na universidade: reflexões a partir do trabalho de supervisão. Psicologia clínica, Rio de Janeiro, v.22, n.2, p.45-55, 2010. Disponível em: <http://www.scielo.br/scielo.php?script=sci_arttext\&pid=S010356652010000200004\&lng=en\&nrm=iso>. Acesso em: 12 jul. 2015.

POPPE, A. R. S.; BATISTA, S. H. S. S. Formação em psicologia no contexto das diretrizes curriculares nacionais: uma discussão sobre os cenários da prática em saúde. Psicologia: ciência e profissão, Brasília, v.32, n.4, p.986-999, 2012. Disponível em: $<$ http://www.scielo.br/scielo.php?script=sci_arttext\&pid=S141498932012000400016\&lng=en\&nrm=iso>. Acesso em: 22 jul. 2015.

RASERA, E. F.; OLIVEIRA, F. M.; JESUS, M. J. B. S. A prática grupal e o discurso sobre a ética em documentos oficiais. Estudos de psicologia (Campinas), Campinas, v.31, n.3, p.405-414, set. 2014. Disponível em: $<$ http://www.scielo.br/scielo.php?script=sci_arttext\&pid=S0103-

166X2014000300009\&lng=en\&nrm=iso>. Acesso em: 22 jul. 2015.

ROCHA, W. B. et al. Percepção de profissionais da saúde sobre abortamento legal. Revista Bioética, Brasília, v.23, n.2, p.387-399, ago. 2015. Disponível em: $<$ http://www.scielo.br/scielo.php?script=sci_arttext\&pid=S 198380422015000200387\&lng=en\&nrm=iso>. Acesso em: 14 jul. 2015.

SANTOS, C. B. et al. A diversidade sexual no ensino de psicologia. O cinema como ferramenta de intervenção e pesquisa. Sexualidad, Salud y Sociedad (Rio de Janeiro), Rio de Janeiro, n.7, p.127-141, abr. 2011. Disponível em: $<$ http://www.scielo.br/scielo.php?script=sci_arttext\&pid=S198464872011000200006\&lng=en\&nrm=iso>. Acesso em: 14 jul. 2015.

SEIXAS, P. S. et al. Projeto pedagógico de curso e formação do psicólogo: uma proposta de análise. Psicologia Escolar e Educacional, Maringá, v.17, n.1, p.113122, jun. $2013 . \quad$ Disponível em: <http://www.scielo.br/scielo.php?script=sci_arttext\&pid=S141385572013000100012\&lng=en\&nrm=iso>. Acesso em: 12 jul. 2015.

SILVA, N. P. Valores priorizados por estudantes universitários de um curso de psicologia de uma universidade pública. Educação e Pesquisa, São Paulo, v.41, n.2, 
p.391-407, jun. 2015. Disponível em:

$<$ http://www.scielo.br/scielo.php?script=sci_arttext\&pid=S1517-

97022015000200391\&lng=en\&nrm=iso>. Acesso em: 22 jul. 2015.

SOUTO, T. S.; BATISTA, S. H.; ALVES BATISTA, N. A educação interprofissional na formação em psicologia: olhares de estudantes. Psicologia: ciência e profissão, Brasília, v.34, n.1, p.32-45, mar. 2014. Disponível em: $<$ http://www.scielo.br/scielo.php?script=sci_arttext\&pid=S1414-

98932014000100004\&lng=en\&nrm=iso>. Acesso em: 22 jul. 2015.

SOUZA, C. R.; COELHO, D. M. O neutro em psicanálise: da técnica à ética. Fractal,

Revista de Psicologia, Rio de Janeiro, v.24, n.1, p.95-110, abr. 2012. Disponível em: $<$ http://www.scielo.br/scielo.php?script=sci_arttext\&pid=S1984-

02922012000100007\&lng=en\&nrm=iso>. Acesso em: 22 jul. 2015.

TEIXEIRA, D. V. Desigualdade de gênero: sobre garantias e responsabilidades sociais de homens e mulheres. Revista Direito GV, São Paulo, v.6, n.1, p.253274, jun. 2010. Disponível em: <http://www.scielo.br/scielo.php?script=sci_arttext\&pid=S180824322010000100012\&lng=en\&nrm=iso>. Acesso em: 22 jul. 2015.

TOLEDO, L. G.; PINAFI, T. A clínica psicológica e o público LGBT. Psicologia clínica, Rio de Janeiro, v.24, n.1, p.137-163, 2012. Disponível em: $<$ http://www.scielo.br/scielo.php?script=sci_arttext\&pid=S0103-

56652012000100010\&lng=en\&nrm=iso>. Acesso em: 22 jul. 2015.

VAHLE, M. A.; CUNHA, E. L. Matrizes clínicas e ética em Freud. Psicologia clínica, Rio de Janeiro, v.23, n.1, p.203-220, 2011. Disponível em: $\quad<$ http://www.scielo.br/scielo.php?script=sci_arttext\&pid=S0103$56652011000100013 \& \operatorname{lng}=\mathrm{en} \& n \mathrm{~nm}=\mathrm{iso}>$. Acesso em: 22 jul. 2015.

VILELA, A. M. J. História da psicologia no Brasil: uma narrativa por meio de seu ensino. Psicologia: ciência e profissão, Brasília, v.32, n.spe, p.28-43, 2012. Disponível em: <http://www.scielo.br/scielo.php?script=sci_arttext\&pid=S1414$98932012000500004 \& \operatorname{lng}=$ en\&nrm=iso $>$. Acesso em: 22 jul. 2015.

ZOTARELI, V. et al. Gender and sexual violence among students at a brazilian university. Revista Brasileira de Saúde Materno Infantil, Recife, v.12, n.1, p.37-46, mar. 2012. Disponível em: <http://www.scielo.br/scielo.php?script=sci_arttext\&pid=S 1519$38292012000100004 \& \operatorname{lng}=$ en\&nrm=iso>. Acesso em: 22 jul. 2015. 